\title{
Advances in Adjuvant and Primary Systemic Therapy of Early Breast Cancer
}

\author{
Joachim Bischoff Serban-Dan Costa \\ Universitätsfrauenklinik Magdeburg, Germany
}

\section{Key Words}

Trastuzumab - Therapy, dose-dense - Therapy, adjuvant · Therapy, primary systemic · Breast cancer

\section{Summary}

The BCIRG (Breast Cancer International Research Group) 006 and FinHER (Finland Herceptin) trials confirm the results of recently published randomized phase III studies that the addition of trastuzumab to standard chemotherapy improves disease-free survival of patients with HER2-positive breast cancer. Moreover, they included 2 different strategies that seem to reduce trastuzumab-associated cardiotoxicity. However, data are preliminary. Dose-dense chemotherapy can be offered to patients with high-risk HER2-negative breast cancer, based on an updated analysis of the CALBG (Cancer and Acute Leukemia Group B) C9741 trial. Nevertheless, sequential FEC (5-fluorouracil/epirubicin/cyclophosphamide)taxane regimens should be considered as adjuvant chemotherapy for node-positive breast cancer, since FEC and docetaxel have been shown to be superior to a standard FEC therapy. Using paclitaxel as taxane compound is an alternative strategy that needs to be re-evaluated after a longer follow-up period. The combination of docetaxel and cyclophosphamide is restricted to patients in whom anthracyclines are contraindicated and who are at intermediate risk of disease recurrence. An extended adjuvant therapy with letrozole is an option for patients who have completed 5 years of tamoxifen. It can be assumed that patients benefit from letrozole even after a longer period of time since the tamoxifen therapy.

\author{
Schlüsselwörter \\ Trastuzumab - Therapie, dosisdichte - Therapie, adju- \\ vante $\cdot$ Therapie, primär systemische $\cdot$ Mammakarzinom
}

\section{Zusammenfassung}

Die Studien BCIRG (Breast Cancer International Research Group) 006 und FinHER (Finland Herceptin) bestätigen die Ergebnisse kürzlich publizierter Phase-III-Studien, dass die Zugabe von Trastuzumab zu einer Standardchemotherapie das erkrankungsfreie Überleben von Patientinnen mit frühem, HER2-positiven Mammakarzinom verbessert. Darüber hinaus verfolgen beiden Studien verschiedene Strategien, die möglicherweise zu einer Reduktion der trastuzumabassoziierten Kardiotoxizität führen. Diesbezüglich bleiben weitere Ergebnisse abzuwarten. Bei fehlender HER2-Überexpression können Hochrisikopatientinnen von einer dosisdichten, adjuvanten Chemotherapie profitieren, basierend auf der aktuellen Auswertung der CALBG (Cancer and Acute Leukemia Group B) C9741-Studie. Bei axillärem Lymphknotenbefall kommt außerdem der Einsatz sequentieller FEC (5-Fluorouracil/Epirubicin/Cyclophosphamid)-Taxan-Protokolle in Frage, da sich die sequentielle FEC/Docetaxel-Therapie gegenüber einem Standard-FEC-Schema als überlegen erwies. Auch der Stellenwert von Paclitaxel in sequentieller Kombination mit FEC wurde überprüft, bleibt jedoch noch im Rahmen weiterer Auswertungen nach längerem Follow-up zu klären. Eine adjuvante Docetaxel/ Cyclophosphamid-Therapie stellt eine Behandlungsalternative für Patientinnen mit einer Kontraindikation gegen Anthrazykline und intermediärem Rezidivrisiko dar. Die erweiterte adjuvante Therapie mit Letrozol ist eine Option für Patienten nach 5-jähriger Tamoxifentherapie. Es ist davon auszugehen, dass sie von diesem Vorgehen auch profitieren, wenn ein längeres Intervall zwischen beiden Behandlungsschritten liegt.

\begin{tabular}{ll}
\hline KARGER & ( 2006 S. Karger GmbH, Freiburg \\
Fax +49 761 4520714 & Accessible online at: \\
$\begin{array}{l}\text { E-mail Information@Karger.de } \\
\text { www.karger.com }\end{array}$ & www.karger.com/brc
\end{tabular}

Dr. med. Joachim Bischoff

Universitätsfrauenklinik der Medizinischen Fakultät Otto-von-Guericke-Universität

Gerhart-Hauptmann-Straße 35, 39108 Magdeburg

Tel. +49 3916717-324, Fax -430

E-mail joachim.bischoff@medizin.uni-magdeburg.de 


\section{Adjuvant Chemotherapy}

In the last decades, the most important progress in the therapy of breast cancer was achieved by the use of adjuvant chemotherapy and endocrine therapy. Recently, an update of the meta-analysis of the early breast cancer trialist collaborative group (EBCTCG) [1] was published confirming the prolongation of survival in patients with primary breast cancer even after a follow-up of 15 years. Thus, anthracycline-based polychemotherapy reduces the annual breast cancer death rate by $20-38 \%$, depending on the age at presentation but not on the use of additional endocrine therapy and tumor-associated prognostic factors. Interestingly, in postmenopausal, estrogen receptor-positive $(\mathrm{ER}+)$ breast cancer, adjuvant tamoxifen reduces the annual breast cancer death rate by $31 \%$, and this effect does not depend on age, use of chemotherapy or other tumor characteristics. Since the data of the EBCTCG meta-analyses rely on well established but 'old' chemotherapy regimens and tamoxifen, we expect further improvements in long-term survival through use of newer drugs or better use of older drugs.

At the San Antonio Breast Cancer Symposium (SABCS) 2005 , data on adjuvant therapy of early breast cancer played a cardinal role. In the present paper, some of these data are reported in brief. Formally, the PACS (Penn Abramson Cancer Center) 01 trial has shown the superiority of the taxane-containing sequential regimen over a standard 5-fluorouracil/ epirubicin/cyclophosphamide (FEC) therapy. According to this design, the GEICAM group (Spanish Breast Cancer Research Group) [2] compared the 2 strategies of adjuvant treatment in patients with node-positive breast cancer. Using paclitaxel as taxane compound after a median follow-up of 47 months, a significant improvement of disease-free survival in favor of the sequential therapy was observed (86.9 vs. $79.2 \%$ ). So far, no statistical differences between the 2 arms regarding overall survival have been reached yet.

An open question in the adjuvant chemotherapy of breast cancer is the role of non-anthracycline-based regimens. Between 1997 and 1999, a randomized phase III trial was performed comparing a docetaxel/cyclophosphamide (TC) regimen with standard doxorubicin/cyclophosphamide (AC). A total of 4 cycles were administered in each arm. The majority of patients were hormone receptor-positive $(\mathrm{HR}+)$ and nodenegative or had a limited involvement of axillary lymph nodes. The data of the final analysis revealed a better disease-free survival for TC after 5 years ( 86 vs. $81 \%, \mathrm{p}=0.027$ ). Moreover, there is a trend towards prolonged overall survival in favor of TC which was the better tolerated regimen [3]. From the epidemiological point of view, one important question is the time course of mortality in breast cancer. This was addressed by Olivotto et al. [4] who analyzed the British Columbia Cancer Registry data of breast cancer specific survival (BCSS) for patients diagnosed in 1995. Absolute 10-year BCSS improved by $20 \%$ compared to 1975 and has subse- quently plateaued. Adjuvant systemic therapy contributed by $6.1 \%$ to this gain.

Recently, the interim analysis of 3 prospective randomized trials showed that adding trastuzumab to adjuvant chemotherapy reduces the risk of relapse in patients with HER2-positive breast cancer by more than $50 \%$. However, if patients received anthracycline-containing regimens and trastuzumab, a significant increase of cardiotoxicity occurred $[5,6]$. Since cardiotoxicity was also associated with simultaneous administration of paclitaxel and trastuzumab, the BCIRG Group (Breast Cancer International Research Group) [7] has carried out a study (BCIRG 006) with docetaxel instead of paclitaxel (therapy arm AC-T: doxorubicin + cyclophosphamide followed by docetaxel (Taxotere ${ }^{\circledR}$, Aventis, Bad Soden, Germany) versus therapy arm AC-TH: AC-T with trastuzumab (Herceptin ${ }^{\circledR}$, Hoffman-La Roche, Grenzach-Whylen, GermanyA)), and also including a non-anthracycline-based study arm containing docetaxel, cis- or carboplatinum and trastuzumab (TCH). The primary endpoint of the BCIRG 006 study was disease-free survival with $80 \%$ power to detect an absolute difference of $7 \%$. Secondary objectives included overall survival and safety (e.g. cardiotoxicity). The first planned analysis was conducted after 322 events, with a median follow-up of 23 months. Both AC-TH and TCH significantly improved disease-free survival in comparison to AC-T (hazard ratio $=0.49$ for AC-TH, 0.61 for $\mathrm{TCH}$ ). There was no statistically significant difference between the 2 trastuzumab-containing regimens, perhaps due to the small number of events. The rate of cardiac events was increased in AC-TH (2.62\%) compared to AC-T (0.86\%, $\mathrm{p}=0.0024)$ but not in TCH $(1.04 \%, \mathrm{p}=0.82 \%)$. There also was a significantly higher incidence of asymptomatic and persistent left ventricular ejection fraction (LVEF) decrease in the AC-TH arm. At present, there is insufficient information to evaluate the secondary endpoint of overall survival [5]. An additional observation was that HER2-positive patients with no co-amplification of the topoisomerase II $\alpha$ gene (approximately $65 \%$ ) do not appear to benefit from anthracycline/ trastuzumab-containing regimens and may be ideal candidates for non-anthracycline-based regimens, thus avoiding potential cardiotoxicity. Longer follow-up is needed to confirm these very interesting data.

Another open question is the optimal length of trastuzumab treatment in the adjuvant setting. Previously published data suggest that HER2-positive patients benefit if trastuzumab is administered over a period of 1 year [3,4] after standard chemotherapy or concomitantly to paclitaxel. The FinHER (Finland Herceptin) study investigated short-term treatment with trastuzumab of only 9 weeks concurrent with 3 3-weekly cycles of docetaxel or 8 weekly cycles of vinorelbine, both followed by 3 3-weekly cycles of FEC. 232 of 1,010 women recruited showed erbB2 amplification and underwent a second randomization to either receive trastuzumab or not. Trastuzumab was effective in preventing any breast cancer recurrence $(11 / 151$ versus $26 / 116$, hazard ratio $0.46, p=0.0078)$. The 
3-year distant disease-free survival was $93 \%$ in patients receiving trastuzumab and $76 \%$ in controls $(p=0.0078$, hazard ratio $0.43,11 / 115$ versus $26 / 116$ events). There was a trend towards superior overall survival for trastuzumab-based therapy without clinically relevant cardiotoxicity [8]. These data suggest that 9 weeks of trastuzumab added to chemotherapy might lead to similar survival benefit as the longer (1-year) administration, with the advantage of cardiotoxicity being avoided. Interpretation of the data of the FinHER trial is impeded by the fact that the sample size is small and the followup is very short.

Since anthracycline/taxane-containing chemotherapies have been widely accepted as standard of care in node-positive breast cancer, these drugs have also been integrated in dosedense regimens. A 2-week schedule of doxorubicin, paclitaxel and cyclophosphamide, given concurrently or sequentially was the first to demonstrate improved disease-free survival (hazard ratio $0.47, \mathrm{p}=0.01$ ) and overall survival (hazard ratio 0.69 , $\mathrm{p}=0.013)$ as compared to conventional 3-weekly schedules [9]. The current analysis of this study with longer follow-up of 5 years was presented in San Antonio by Hudis [10]. The greatest benefit was found in the estrogen receptor-negative (ER-) subset. No increased risk of long-term toxicity was observed. The final report of a phase I/II study with sequential dose-dense chemotherapy containing epirubicin, paclitaxel and cyclophosphamide (ETC) was presented in San Antonio by Möbus [11]. After a median follow-up of 6.5 years, diseasefree survival $(73 \%)$ and overall survival $(79 \%)$ correspond with the promising preliminary results of the ETC phase III trial, which were presented in 2004.

Several pilot studies have been conducted to assess the feasibility of docetaxel under dose-dense conditions. Based on the results of PACS 01, the PACS 06 study compared 3 cycles of FEC 100 (5-FU, epirubicin $100 \mathrm{mg} / \mathrm{m}^{2}$, cyclophosphamide), followed by 32 -weekly cycles of docetaxel $100 \mathrm{mg} / \mathrm{m}^{2}$ versus a 4-week schedule as known from the PACS 01 study. After enrollment of 73 patients in each arm, 14 cases of skin and handfoot syndrome grade 3 and 4 were seen during the 2-weekly docetaxel schedule versus 3 cases during the 4-weekly docetaxel treatment. Due to this high incidence of dose-limiting cutaneous toxicities, patient accrual was stopped in arm A [12]. Instead of changing the sequence of administration between doxorubicin and docetaxel in a dose-dense regimen, another phase II trial confirmed the evidence of docetaxel-mediated skin toxicity. Nevertheless, this regimen was feasible.

\section{Adjuvant Endocrine Treatment}

There has been some evidence suggesting a selective responsiveness to aromatase inhibitors in different subsets of HR+ breast cancer. Thus, in the ATAC (Arimidex, Tamoxifen, Alone or in Combination) trial, the benefit of anastrozol over tamoxifen appears to be restricted to ER+/progesteron recep- tor-negative (PR-) patients. In contrast, data from the MA-17 trial suggest that extended adjuvant therapy with letrozole after 5 years of tamoxifen does not improve disease-free survival in this subgroup. These data have to be interpreted cautiously, because subset analysis has not been planned initially and ER/PR measurements were done at several institutions. The results of the central measurement of standard ER and PR levels are ongoing and data are pending [12].

In the MA-17 trial, all patients received tamoxifen for 5 years and were then randomized to letrozole or placebo $[13,14]$. Because of the significant benefit elicited by letrozole, the study was closed and some patients in the placebo arm received subsequently letrozole. An updated analysis of the MA-17 trial post unblinding offers information on 2,247 women originally assigned to placebo [15]. Among them, 601 patients crossed over from placebo to letrozole. These patients had a significant increase in disease-free survival as compared to controls (adjusted hazard ratio 0.53, $\mathrm{p}=005$ ). A further analysis of the patients in the MA-17 study revealed that placebo patients had an increased risk of disease recurrence after discontinuing tamoxifen. In contrast, patients receiving letrozole showed a significantly lower recurrence rate $(p=0.02)$. In order to assess the issue of duration of letrozole therapy, a re-randomization of all participants completing letrozole in the MA-17 trial to a further 5 years of treatment has been started $[16,17]$.

\section{Neoadjuvant Therapy of Primary Breast Cancer}

To date, only few data are available on the role of trastuzumab in the neoadjuvant therapy of HER2-positive breast cancer. Recently, a sequential therapy with paclitaxel followed by FEC combined with trastuzumab showed encouraging results. In this prospective randomized phase III trial, patient accrual was stopped because of the inferiority in the control arm not receiving trastuzumab. 22 additional patients were treated with the experimental combination. A pathological complete remission ( $\mathrm{pCR}$ ) rate of $54.5 \%$ confirmed the promising data published initially $[18,19]$.

Preliminary results of another phase II trial investigating neoadjuvant therapy with docetaxel, carboplatin and trastuzumab were also presented at the SABCS 2005. 44 patients were assessable for efficacy as the secondary endpoint and showed a complete response of $43.3 \%$. Central assessment of the HER2 immunohistochemitry revealed HER2negative tumors in 8 patients (HER2 score $1+$ in 5 patients and $2+$ in 3 patients), and therefore fluorescent in situ hybridization (FISH) will be performed. It can be assumed that this rate of false-positive HER2 determinations has a significant influence on efficacy data. Similar results were found in another study evaluating TCH in the neoadjuvant setting. 55 patients were stratified by HER2 status according to FISH analysis. Paclitaxel and trastuzumab were administered week- 
ly in combination with 4-weekly carboplatin. HER2-positive patients were more likely to achieve pCR $(78 \%, 14 / 18)$ than HER2-negative patients $(29 \%, 10 / 35)$ [20]. In a study presented by Mehta [21], patients $(n=21)$ received 4 cycles of TCH followed by 4 cycles of dose-dense doxorubicin and cyclophosphamide with or without trastuzumab, depending on HER2 overexpression. 5 of 8 HER2-positive patients achieved pCR. This regimen was shown to be feasible and non-toxic, and should be further investigated.

Survival data for patients treated with neoadjuvant trastuzum$\mathrm{ab}$ are not yet available. Therefore, it remains unclear how trastuzumab could be incorporated into the systemic treatment of primary breast cancer. So far, most HER2-positive patients receiving neoadjuvant chemotherapy in combination with trastuzumab were part of the HERA (HERceptin Adjuvant Trial) study. More than $10 \%$ of the 5,090 study patients had received neoadjuvant chemotherapy. Patients were then randomized to either 1 or 2 years of trastuzumab or were observed after surgery (i.e. controls). Promising first results of the HERA trial warrant a prospective randomized trial to compare postoperative with upfront trastuzumab in the neoadjuvant setting.

It has to be stressed that approximately $75 \%$ of the breast cancer patients have HER2-negative breast cancer. In these patients, several neoadjuvant regimens have been evaluated and updated at the SABCS 2006. In one phase II study, 53 patients received dose-dense docetaxel $100 \mathrm{mg} / \mathrm{m}^{2}$ every 2 weeks. Docetaxel therapy was completed in all patients, but skin toxicity was dose-limiting. The achieved $\mathrm{pCR}$ rate of $12 \%$ was quite low and may be due to the high proportion of $\mathrm{HR}+$ cases and the small number of patients treated [22]. In the study presented by Ditsch [23], dose-dense sequential epirubicin/paclitaxel (ET) therapy was superior to conventional ET treatment (pCR 21 vs. 12\%) in terms of pathological response in 101 patients with inflammatory breast cancer. Another important issue in neoadjuvant chemotherapy is prediction of response. Since pCR correlates well with survival, it can be used as a surrogate marker for survival in breast cancer. Therefore, neoadjuvant therapy represents an important tool for testing new therapeutic options. Several biological markers have been shown to predict responsiveness of the tumor to neoadjuvant chemotherapy, but data are still conflicting. In 2 cohort studies, patients with invasive lobular carcinoma seemed to be more responsive to anthracycline-based therapy than patients with invasive ductal carcinoma. However, the subgroup of patients with invasive lobular carcinoma was very small in both studies [24, 25]. In the GEPARTRIO study [26], the only parameter predicting pathological response in a multivariate analysis was the ER and PR status (hazard ratio 4.56, $\mathrm{p}=0.001$ ), but not the tumor size or histological subtype. In this trial, patients were initially treated with 2 cycles of TAC and non-responders were randomized to receive either 4 additional cycles of docetaxel/doxorubicin/cyclophosphamide (TAC) or 4 cycles of vinorelbine/capecitabine (NX). Two thirds of these patients showed a late clinical response, but the pCR rate was very low $(<5 \%)$. The NX regimen was better tolerated than further administration of TAC, but efficacy was similar in both arms.

\section{References}

1 Early Breast Cancer Trialists' Collaborative Group: Effects of chemotherapy and hormonal therapy for early breast cancer on recurrence and 15-year survival: an overview of the randomised trials. Lancet 2005;365:1687-1717.

2 Martin M, Rodríguez-Lescure A, Ruiz A, Alba E, Calvo L, Ruiz-Borrego M, Munárriz B, Lopez-Vega JM, Rodríguez CA, Crespo C: Multicenter, randomized phase III study of adjuvant chemotherapy for node positive breast cancer comparing 6 cycles $\mathrm{FE}_{90} \mathrm{C}$ versus 4 cycles of $\mathrm{FE}_{90} \mathrm{C}$ followed by 8 weekly paclitaxel administrations: interim efficacy analysis of GEICAM9906 Trial. Breast Cancer Res Treat 2005;94(suppl 1):abstr 39

3 Jones SE, Savin MA, Holmes FA, O'Shaughnessy JA, Blum JL, Vukelja SJ, Geroge TK, McIntyre KJ, Pippen JE, Sandbach J, Kirby RL, Bordelon JH, Hyman WJ, Negron AG, Khandewal P, Richards DA, Anthony S, Nugent JE, Mennel RG, Banerji M, Edelman G, Ruxer RL, Amare M, Kampe CE, Koutrelakos N, Meyer WG, Asmar L: Final analysis: TC (docetaxel/cyclophosphamid, 4 cycles) has a superior disease-free survival compared to standard AC (doxorubicin/cyclophosphamid) in 1016 women with early stage breast cancer. Breast Cancer Res Treat 2005;94(suppl 1):abstr 40.
4 Olivotto IA, Coldman AJ, Speers C, Phillips N, Goldrick A, Gelmon KA: Improved breast cancer specific survival (BCSS): the impact of adjuvant systemic therapy. Breast Cancer Res Treat 2005;94 (suppl 1):abstr 2073.

5 Romond Edward H, Perez Edith A, Bryant John, et al.: Trastuzumab plus adjuvant chemotherapy for operable HER2-positive breast cancer. N Engl J Med 2005;353:1673-1684.

6 Piccart-Gebhart MJ, Procter M, Leyland-Jones B, et al.: Trastuzumab after adjuvant chemotherapy in HER2-positive breast cancer N Engl J Med 2005; 353:1659-1672.

7 Slamon D, Eiermann W, Robert N, et al.: Phase III randomized trial comparing doxorubicin and cyclophosphamide followed by docetaxel $(\mathrm{AC} \rightarrow \mathrm{T})$ with doxorubicin and cyclophosphamide followed by docetaxel and trastuzumab $(\mathrm{AC} \rightarrow \mathrm{TH})$ with docetaxel, carboplatin and trastuzumab (TCH) in HER2 positive breast cancer patients: BCIRG 006 study. Breast Cancer Res Treat 2005;94(suppl 1): abstr 1.

8 Joensuu H, Kellokumpu-Lehtinen PL, Bono P, et al.: Adjuvant docetaxel or vinorelbine with or without trastuzumab for breast cancer. N Engl J Med 2006;345:789-790.
9 Citron ML, Berry DA, Cirrincione C, et al.: Randomized trial of dose-dense versus conventionally scheduled and sequential versus concurrent combination chemotherapy as postoperative adjuvan treatment of node-positive primary breast cancer: first report of Intergroup Trial C9741/ Cancer and Leukemia Group B Trial 9741. Breast Cancer Res Treat 2005;94(suppl 1):abstr 7.

10 Hudis C, Citron M, Berry D, et al.: Five years follow-up of INT C9741: dose-dense (DD) chemotherapy (CRx) is safe and effective. Breast Cancer Res Treat 2005;94(suppl 1):abstr 41.

11 Moebus V, Hauser N, Kurbacher C, et al.: Longterm follow-up of sequential dose-dense chemotherapy with epirubicin (E), paclitaxel (T) and cyclophosphamide (C) ETC) in breast cancer patients with 4-9 positive nodes: final results of a phase I/ II study. Breast Cancer Res Treat 2005;94(suppl 1): abstr 2065.

12 Fumoleau P, Brain E, Delozier T, et al.: Adjuvant dose-dense (3 FEC 100 followed by 3 docetaxel every 2 weeks) regimen is not feasible. Results of the FNCLCC-PACS 06 study. Breast Cancer Res Treat 2005;94(suppl 1):abstr 2071.

13 Goss PE, Ingle JN, Massachusetts Tu D, et al.: NCIC CTC MA17: disease free survival according to estrogen receptor and progesterone receptor status of the primary tumor. Breast Cancer Res Treat 2005;94(suppl 1):abstr 2042. 
14 Goss PE, Ingle JN, Martino S, et al.: A randomized trial of letrozole in postmenopausal after five years of tamoxifen therapy for early-stage breast cancer. N Engl J Med 2003;349:1793-1802.

15 Goss PE, Ingle JN, Martino S, et al: Updated analysis of the NCIC CTG MA.17 randomized placebo (P) controlled trial of letrozole (L) after five years of tamoxifen in postmenopausal women with early stage breast cancer. Proc Am Soc Clin Oncol 2004, 23:87(abstr 84).

16 Goss PE, Ingle JN, Palmer MJ, et al.: Updated analysis is NCIC CTG MA.17 (letrozole vs. placebo to letrozole vs. placebo) post unblinding. Breast Cancer Res Treat 2005;94(suppl 1):abstr 16.

17 Ingle JN, Goss PE, Tu D, Mayo Clinic, et al.: Analysis of duration of letrozole extended adjuvant therapy as measured by hazard ratios of disease recurrence over time for patients on NCIC CTG MA.17. Breast Cancer Res Treat 2005;94(suppl 1):abstr 17.

18 Buzdar AU, Ibrahim NK, Francis D, et al.: Significantly higher pathologic complete remission rate after neoadjuvant therapy with trastuzumab, paclitaxel, and epirubicin chemotherapy: results of a randomized trial in human epidermal growth factor receptor 2-positive operable breast cancer. J Clin Oncol 2005:23:3676-3685.
19 Buzdar AU, Valero V, Ibrahim N, et al.: Prospective data of additional patients treated with neoadjuvant therapy with paclitaxel followed by FEC chemotherapy with trastuzumab in HER-2 positive operable breast cancer, and an update of initial study population. Breast Cancer Res Treat 2005;94 (suppl 1):abstr 5049.

20 Fenton MA, Ries LM, Strenger R, et al.: Frequent pathologic complete responses seen with neoadjuvant $\mathrm{q} 4$ week carboplatin and weekly paclitaxel \pm weekly trastuzumab in respectable and locally advanced breast cancer: a Brown University Oncology Group (BrUOG) Study. Breast Cancer Res Treat 2005;94(suppl 1):abstr 5054.

21 Mehta RS, Schubbert T, Hsiang D, et al.: High pathological complete remission rates with paclitaxel and carboplatin \pm trastuzumab $(\mathrm{TC} \pm \mathrm{H})$ following dose-dense doxorubicin and cyclophosphamid (AC) supported by GM-CSF in breast cancer-a phase II study. Breast Cancer Res Treat 2005; 94(suppl 1):abstr 5056.

22 García-Mata J, Calvo L, García-Palomo A, et al.: Dose-dense taxotere $(\mathrm{T})$ and adriamycin/ cyclophosphamide (AC) as neoadjuvant treatment in locally advanced breast cancer (LABC). Breast Cancer Res Treat 2005;94(suppl 1):abstr 5059.
23 Ditsch N, Bauerfeind I, Kahlert ST: Prospective randomized comparison of neoadjuvant/primary systemic therapy (PST) with dose-dense sequential epirubicin (E)/paclitaxel (P) chemotherapy versus standard dose therapy regimen in patients with breast cancer (bc), multicentre study of the AGO, Germany): subgroup analysis of inflammatory bc. Breast Cancer Res Treat 2005;94(suppl 1):abstr 5051.

24 Tubiana-Hulin MJ, Stevens DM, Guinebretiere JMR, et al.: Response to primary chemotherapy in breast carcinoma depends on histological tumor type: a study on 860 patients from one institution. Breast Cancer Res Treat 2005;94(suppl 1):abstr 5063.

25 Vincent-Salomon A, Pierga JY, Gautier C, et al. Neoadjuvant chemotherapy for invasive lobular carcinomas of the breast: a poorer response rate but not a worse prognosis than invasive ductal carcinoma. Breast Cancer Res Treat 2005;94(suppl 1): abstr 5071.

26 Von Minckwitz G, Blohmer JU, Loehr A, et al. Comparison of docetaxel/ doxorubicin/ cyclophosphamide (TAC) versus vinorelbine/ capecitabine (NX) in patients non-responding to 2 cycles of neoadjuvant TAC chemotherapy-first results of the phase III GEPARTRIO study by the German Breast Group. Breast Cancer Res Treat 2005;94 (suppl 1):abstr 38. 